\title{
A PHANTOM SUPERNUMERARY LIMB AFTER A CERVICAL ROOT LESION
}

\author{
Macdonald Critchley
}

We nowadays realize that after amputations of a limb it is the rule and not the exception for phantom experiences to follow. Indeed, it is only in the case of "congenital" amputations, or loss of a limb in very early life, that these phantoms do noz ordinarily occur. Less is known of the existence of phantom sensations after what has been called "biological" amputations, that is, loss of motor and sensory innervation, leaving the mass of a limb intact, but paralysed and insensitive. 'There is evidence, none the less, that in some cases of cerebral hemiplegia, the patient's mental impression as to the attitude and physical qualities of the paralysed limb, are quite different from the actual state of affairs. In other words, the subject may have a sort of "phantom third limb" in addition to his paralysed extremity. Such phantoms are far from being common, though close questioning will no doubt reveal a number of unsuspected instances. Phantom third limbs are probably found more often after lesions of the subordinate than of the dominant hemisphere. The site of the responsible focus of ảisease is probably both deep and posterior, for hemianaesthesia and hemianopsia usually co-exist.

Phantom sensations may also follow spinal lesions. Since it is necessary for the resulting motor and sensory loss to be severe, the best instances are to be met with in the examples of paraplegia following injuries to the cord, or perhaps spinal tumour, or myelitis. Bors has recently described a series of 50 such cases. Even in lesions of the conus medullaris, phantom sensations may occur, but in such cases it is the anus and not the extremities which occupy a pathological part in the body-image. Riddoch has described phantom feelings in one leg after a spinal cord lesion involving mainly the posterior columns. This same author has emphasized the rarity of phantom sensations after peripheral nerve lesions. Indeed, the converse phenomenon ("negative phantom") seems to occur at times, for Head's case is quoted of a soldier who sustained an ulnar nerve injury, and later had to have the arm removed; in the resulting phantom limb, the little finger was not represented. L. van Bogaert and Lurje (1936) have, however, described phantom feelings of additional limbs in two cases of polyneuritis.

It would seem that phantom sensations are also to be expected after lesions involving spinal roots or plexuses. Thus Hecaen, David and Talairach (1945) described phantom lower limbs appearing after a compres- 
sion of the cauda equina. Riddoch (1941) declared he had never met with a phantom phenomenon after lesions of the lumbo-sacral plexus. He referred briefly to examples following extensive damage to the brackial plexus. He gave details of one case where the patient, who had no motor or sensory function below his elbow, neveriheless had painful phantom fingers, hand and forearm which he fancied he could inove, situated in alignment with his real limb. After operative exploration of the plexus, the phantom became to some extent separated from the actual limb, and when the latter was moved passively, the phantom remained immobile. One year later, the paralysed arm was amputated; the phantom persisted but gradually underwent the usual process of telescopirg Riddoch also referred to phantom sensations following severe disease of the posterior roots (tabes; hypertrophic polyneuritis).

Hasenjäger and Pötzl (1941) gave a full account of phantom sinsations after an injury to the brachial plexus. The actual limb would be lying impoient upon the bedclothes in an extended attitude, but the patient would distinctly feel a flexed phantom arm lying across his chest, the fingers being bent. The most vivid parts of the phantom were the distal poriions which appeared to be of normal dimensions. If the patient passively moved his paralysed limb so as to make it coincide with the position of the phantom, the latter would disappear. In this particular case, the actual limb was not only insensitive but also painless, while the phantom was at times the seat of sharp, though fleeting painful feelings. The authors mentioned that a few similar cases had been previously recorded. One was an instance of a compression of the roots by a tumour; another was a case of contusion of the brachial plexus. $O$. Foerster and also Gagel, according to these same authors, had experienced the phenomenon of a phantom arm appearing after operative section of the posterior roots of $\mathrm{C}_{4}$ to $\mathrm{D}_{3}$, necessary to gain access to a spinal tumour. Probably the first recorded case of a phantom limb after an injury to the plexus was made by Mayer-Gross (1929).

The common feature in all these cases is the occurence of phaniom sensations in cases when a limb is not "lost" (i.e. surgically removed), but is still present, though rendered functionless hy injury or disease of the nerve-supply. The phantom sensation then constitutes a "phantom third limb", or even "phantom fourth limbs" as in the paraplegic cases. Perhaps the term "phantom supernumerary limb" would best describe these imaginary limbs which are associated with hemiplegia, paraplegia, and also lesions of the roots and plexuses. Characteristic of all these phantom supernumerary limbs is the occasional or constant non-correspondence of the phantom and real limbs in attitude or size. Passive manipulations of the real limb may cause the phantom feeling to move; or the two may in this manner be caused to merge. In this way, the phantom may be regarded as having temporarily disappeared. The phantom limb may be painful, which the actual paralysed limb is not. Further interference with 
the inadequate nerve supply to the limb, as after operative exposure, may cause some alteration in the nature of the phantom, and subsequent removal of the diseased limb may again be followed by a change in the qualities of the phantom.

As Hasenjäger and Pötzl pointed out, the psychological processes at work entail a disharmony between the proprioceptive factors and the visual impressions in the maintenance of the body-image. The former cease to play any active part while the latter are still operative. This discrepancy leads to a sort of imaginal diplopia, in which the false inage - produced by incongruous kinaesthetic meinories - constitutes the phantom limb. It would seem that when there is a rivalry, or lack of correspondence, between visual and sensory components in the body-image, the latter dominate and the false sensory image is accepted, rather than the true visual one. Whether this is a universal state of affairs is perhaps open to debate. The frequency with which phantom sensations follow lesions of the spinal roots and plexus is still not known; that is, whether indeed they are to be regarded as exceptional or as the rule. The day-to-day change in vividness of the spectral limb is another phenomenon which suggests the interplay of fluctuating visual and sensory factors, fluctuating, that is, according to a wide range of endogenous and exogenous circumstances. Herein may be mentioned factors such as the time of day, fatigue, temperature, drow. siness of wakefulness, attention. It is obvious that the variants are made up of both mental and physical conditions, some of which may be bound up closely with the total personality of the patient.

D. D., a young man of 22 years, set out on his motor cycle early in the morning of the 14th June, 1951. His memory from the time of taking breakfast was a blank until he recovered consciousness in the local vicarage about 30 minutes later. During the intervening period he had crashed on his cycle with a motor car and had sustained multiple injuries, including a concussion. He was taken to hospital, where he remained muddled for the next 12 or 24 hours. For the most part of the first three days in hospital he slept. Gradually, as he became more alert he realized that his right arm hurt him a great deal, and that he could not move it. In addition, a feeling of numbness was present from the right shoulder, arm, forearm, and the thumb and index finger and the outer side of the right hand. This loss of power and of sensation in the right upper extremity persisted even after his symptoms of concussion had worn off. In addition, he noticed that the palm of the right hand tended to sweat excessively, and that the palm was hyper-sensitive to touch and to warmtin.

The patient first consulted me on September 18th, 1951. He showed considerable weakness and wasting of the right arm, together with hyperhidrosis of the palm, and sensory loss mainly in the territory of $\mathrm{C}_{4}, \mathrm{C}_{5}$ and $\mathrm{C}_{6}$. It was considered probable that he had sustained a lesion of the outer cord of the brachial plexus.

On October 15th, 1951, he was admitted to the National Hospital, Queen Square, London (Case No. 33383). General examination was carried out by Drs. C. Kalanova and $R$. Hierons and proved negative, including the mental state. Clinical testing of the nervous system revealed a mild oculo-sympathetic palsy on the right side. There was a weakness and some atrophy of the trapezius 
and sterno-mastoid muscles on the right. 'The right arm lay at the side, internally rotated at the shoulder, with the elbow extended and the forearm pronated. Wasting was present in the levator scapulae, supra and infra-spinati, part of the pectoralis major, deltoid; triceps, biceps, forearm muscles and the hypothenar eminence. All movements of the right upper limb and hand were grossly impaired, the following being best retained. pronation of the forearm, flexion of the wrist and of the fingers, abauction of the 2nd, 3rd and 4th fingers, abduction of the thumb and some flexion of the distal phalanx. 'Ionus was decreased in the right arm. No fibrillation or fasciculation was visible. The biceps, triceps and supinator jerks were absent. Sensory testing revealed an area of loss to light touch (cot-

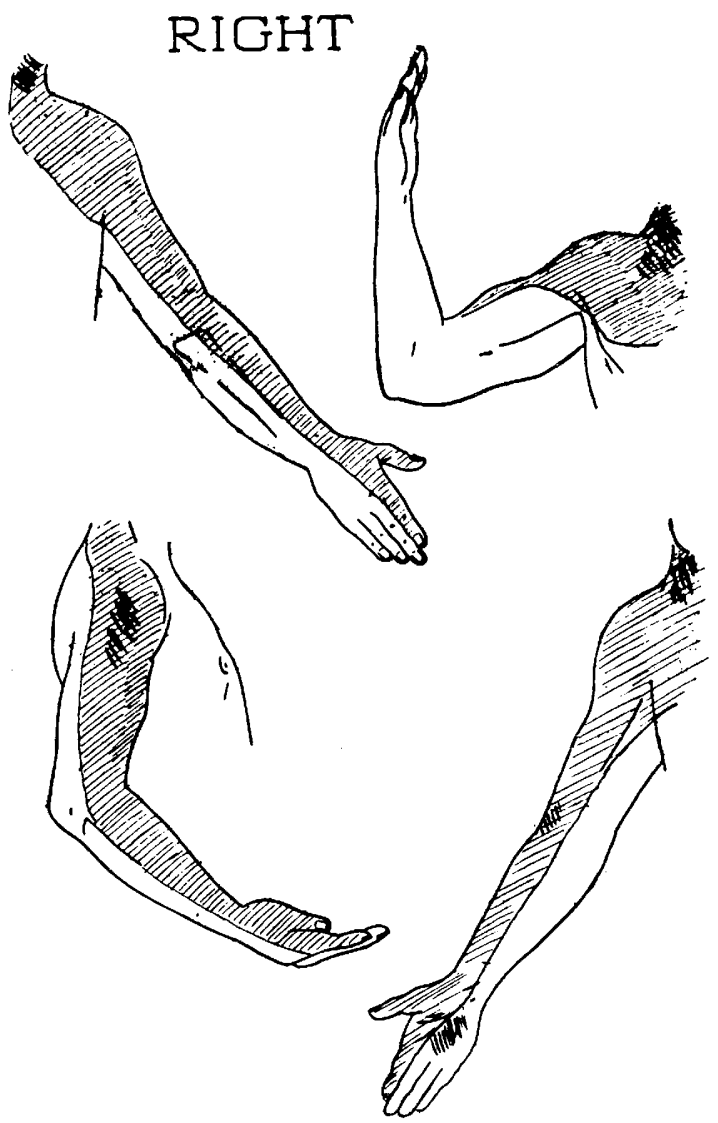

Fig. 1 - In coarse hatching, tenderness on deep pressure, and hyperaesthesia of the palm. In thin hatching, loss to cottonwool, pain felt merely as "touch", loss to cold, hot stimuli felt after a delay merely as warm, loss of position sense in first finger and thumb.

tonwool), pain (pin-prick) and temperature over the outer side of the neck on the right, the right shoulder region, the right arm, forearm, thumb and outer half of the index. Passive movements in the thumb and index were not well appreciated (fig. 1). Deep pressure upon the right upper arm and forearm, and over the right side of the neck, evoked some pain. Active and passive rotation of the neck to the left was limited and uncomfortable. 'The fingers of the right hand were cold and slightly cyanotic. Skiagrams of the cervical ver- 
tebrae revealed no abnormality, but an X-ray picture of the right clavicle showed a fracture-line through the middle shaft, without any considerable displacement. An electromyogram gave evidence of complete denervation of the muscles of the right upper limb, except in the case of the pectoralis major. Here there was a moderate amount of spontaneous activity, but on voluntary contraction a reduction in motor unit activity was not sufficient to be recognizable.

The question of whether or not the neck should be explored was discussed, but the opinion of the neuro-surgeon and of two orthopaedic surgeons was that no useful purpose was likely to be served by any such operative measure.

It was considered probable that the symptoms and signs were due not strictly to a brachial plexus injury, but rather to an avulsion of the anterior roots of $\mathrm{C}_{3}$ to $\mathrm{C}_{7}$ and the posterior roots of $\mathrm{C}_{3}$ to $\mathrm{C}_{6}$ from the spinal cord.

Phantom manifestations - 'The patient remembered that persisting throughout the first 10 days or so after the injury, he had had a very strong impression that his paralysed arm lay along his right side (actually it was immobilized in a splint in an attitude of abduction to $90^{\circ}$ ). It seemed as though he could move this phantom limb in the noma! way, especially the hand. When the surgeons moved his paralysed arm passively: “... it didn't feel like me at all”. Moreover, he went on to say: "It didn't look like my own arm, but more like the limb of a puppet, or someone else's arm... As far as I was concerned, it did not belong to me; it did not look like mine... I was convinced that mine was by my side".

About 10 days after the accident, the phaniom third limb began to disappear, or rather, to merge with the actual paralyzed limb. This was first noted during the surgeon's handling of the paralysed limb. The patient at the same time "willed" the phantom limb to move, whereupon the phantom adopted the same posture as the actual limb, and the phanton feeling gradually disappeared.

'There were occasions, however, when even later, awareness of the paralysis was temporarily suspended. Thus, he dreamt that he was riding his motor cycle as of old, with two normal hands and arms. Occasionally on waking in the mornings, he would feel as he did before the accident, that is, as if he had the full use of his limbs. But on attempting to move the right $\mathrm{arm}_{3}$ he would then realize that it was useless.

Once, while he was in hospital, he felt for a short time on waking out of sleep as if he had two upper arms, but as if the two forearms had become fused at the elbows so as to form a single impaired forearm (apparently this was the left forearm). Immediately he moved the left forearm, however, the impression of fusion disappeared.

\section{DISCUSSION}

It needs to be discussed whether, in the traumatic cases at any rate, more centrally placed lesions even than the plexus or coots may not be taking a part in the genesis of phantom supernumerary sensations. Thus, in the patient described in this paper, it might be argued that the head injury sustained at the same time as the root avulsion, nay have been more serious than at first suspected. Could a coincidental contusion of the parietal lobe, either on the right or on the left side, be exercising an influence upon the morbid manifestations of the body-image? It is 
interesting to note that Hasenjäger and Pötzl also raised the same query, and they could not absolutely exclude the possibility of an attendant cerebral commotio in their case. Mayer-Gross' patient also sustained a head injury with a short period of unconsciouness. After the accident, he developed a post-concussional state with conspicuous changes in his personality.

In the present case-record it can be asserted that in the production of phantom sensations, we are witnessing an interaction at least two levels of the nervous system. There is the negative outfall factor produced by the root lesions; there are the positive manifestations produced at a cerebral, perhaps even cortical, level. Whether it is necessary to postulate that these latter are in part at any rate the evidence of organic dysfunction is open to argument. Certainly in this present case no other clinical or neuropsychological evidence was forthcoming to suggest a parietal dysfunction, even after specific and scrupulous searching. Electro-encephalography was deliberately carried out in an endeavour to detect minor changes in electrical potential over either hemisphere. A good deal of abnormal, slow activity was demonstrated, but symmetrically so over the two hemispheres.

On the other hand, the patient's attitude towards his diseased limb, during the first ten days after the accident, was not altogether normal. His statement that: "... it did not look like my own arm, but more like the arm of a puppet, or someone else's arm", is strongly reminiscent of the defective awareness of paralysis seen in some cases of right parietal disease (Babinski's anosognosia). The reaction is even nore morbid in its quality in that the patient was tending to confabulate or explain away the paralysis, by rejecting the idea that the limb belonged to him, in favour of the view that it was inanimate, or perhaps even the property of someone else. This is, of course, the "identification anosognosia" of Juba. The patient's final statement: " $\ldots$ as far as I was concerned it did not belong to me; it did not look like mine... I was convinced that mine was by my side", virtually amounts to a denial of the existence of disease, the "somatoparaphrenia" of Gerstmann.

These statements on the patient's part, coupled with the fact that the disorders of the body-image appeared soon after consciousness was restored and lasted for ten days in most vivid fashion, suggest the operation of an abnormal parietal lobe. There is no evidence to indicate whether this commotio was unilateral or generalized.

It is perhaps fair to conclude that, while supernumerary phantom limbs may follow lesions of the peripheral nervous system (roots, plexus), in traumatic cases, a coincidental, though perhaps transitory, cerebral 
damage should always be suspected. This factor should be particularly looked for, if the phantom feelings are most vivid during a period of clouded consciousness or delusion; or if any suggestion is present in the nature of denial of the paralysis, or rejection of the owncrship of the affected limb, or confabulatory identification of the limb with other in. dividuals or with inanimate objects.

\section{REFERENCES}

Bors, E. - Arch. Neurol. a. Psychiat., 66:610, 1951.

Hecaen, H., David, M. and 'Talairach, J. - Rev. Ncurol., 78:146, 1945.

Riddoch, G. - Brain, 64:197, 1941.

Hasenjäger, 'Th. and Pötzl, O. - Deutsche Ztschr. f. Nervenh., 152:112, 191.1.

Foerster, O. - Nova Acta Leopoldina (I Ialle), 3, Nr. 10.

Mayer-Gross, W. - Nervenarzt, 2:65, 1929.

National Hospital, Queen Square - London, England. 